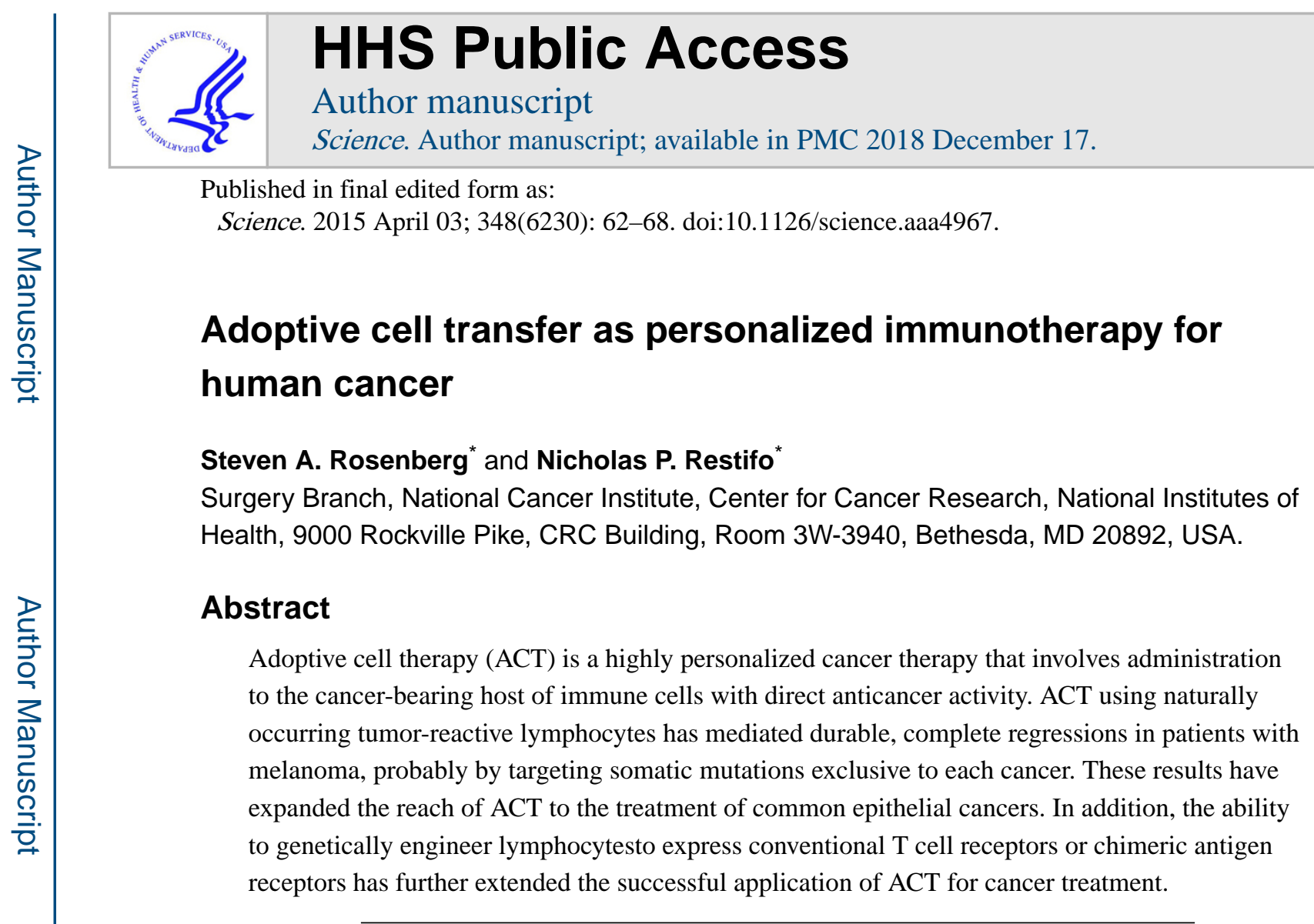

Adoptive cell therapy (ACT) has multiple advantages compared with other forms of cancer immunotherapy that rely on the active in vivo development of sufficient numbers of antitumor T cells with the functions necessary to mediate cancer regression. For use in ACT, large numbers of antitumor lymphocytes (up to $10^{11}$ ) can be readily grown in vitro and selected for high-avidity recognition of the tumor, as well as for the effector functions required to mediate cancer regression. In vitro activation allows such cells to be released from the inhibitory factors that exist in vivo. Perhaps most importantly, ACT enables the manipulation of the host before cell transfer to provide a favorable micro-environment that better supports antitumor immunity. ACT is a "living" treatment because the administered cells can proliferate in vivo and maintain their antitumor effector functions.

A major factor limiting the successful use of ACT in humans is the identification of cells that can target antigens selectively expressed on the cancer and not on essential normal tissues. ACT has used either natural host cells that exhibit antitumor reactivity or host cells that have been genetically engineered with antitumor T cell receptors (TCRs) or chimeric antigen receptors (CARs). With the use of these approaches, ACT has mediated dramatic regressions in a variety of cancer histologies, including melanoma, cervical cancer, lymphoma, leukemia, bile duct cancer, and neuroblastoma. This Review will discuss the current state of ACT for the treatment of human cancer, as well as the principles of effective treatment that point toward improvements in this approach.

exclusive licensee American Association for the Advancement of Science. No claim to original U.S. Government Works *Corresponding author. sar@nih.gov (S.A.R.); restifo@nih.gov (N.P.R.). 


\section{A brief history of ACT}

Very little was known about the function of T lymphocytes until the 1960s, when it was shown that lymphocytes were the mediators of allo-graft rejection in experimental animals. Attempts to use $\mathrm{T}$ cells to treat transplanted murine tumors were limited by the inability to expand and manipulate $\mathrm{T}$ cells in culture. Thus, ACT used transfer of syngeneic lymphocytes from rodents heavily immunized against the tumor, and modest growth inhibition of small established tumors was observed $(1,2)$. In early preclinical studies, the importance of host inhibitory factors was suggested by findings that lymphodepletion using either chemotherapy or radiation before cell transfer enhanced the ability of transferred lymphocytes to treat established tumors $(3,4)$.

The ability to use ACT was facilitated by the description of T cell growth factor [interleukin-2 (IL-2)] in 1976, which provided a means to grow T lymphocytes ex vivo, often without loss of effector functions (5). The direct administration of high doses of IL-2 could inhibit tumor growth in mice (6), and studies in 1982 demonstrated that the intravenous injection of immune lymphocytes expanded in IL-2 could effectively treat bulky subcutaneous FBL3 lymphomas (7). In addition, administration of IL-2 after cell transfer could enhance the therapeutic potential of these adoptively transferred lymphocytes (8). The demonstration in 1985 that IL-2 administration could result in complete durable tumor regressions in some patients with metastatic melanoma (9) provided a stimulus to identify the specific $\mathrm{T}$ cells and their cognate antigens involved in this cancer immuno-therapy. Lymphocytes infiltrating into the stroma of growing, transplantable tumors were shown to represent a concentrated source of lymphocytes capable of recognizing tumor in vitro, and studies in murine tumor models demonstrated that the adoptive transfer of these syngeneic tumor-infiltrating lymphocytes (TILs) expanded in IL-2 could mediate regression of established lung and liver tumors (10). In vitro studies in 1986 showed that human TILs obtained from resected melanomas contained cells capable of specific recognition of autologous tumors (11), and these studies led in 1988 to the first demonstration that ACT using autologous TILs could mediate objective regression of cancer in patients with metastatic melanoma (12).

Populations of TILs that grow from tumors are generally mixtures of $\mathrm{CD}^{+}$and $\mathrm{CD} 4^{+} \mathrm{T}$ cells with few if any major contaminating cells in mature cultures. The ability of pure populations of $\mathrm{T}$ lymphocytes to mediate cancer regression in patients provided the first direct evidence that $\mathrm{T}$ cells played a vital role in human cancer immunotherapy. However, responses were often of short duration, and the transferred cells could rarely be found in the circulation just days after administration. A critical improvement in the application of ACT to the treatment of human cancer was reported in 2002 , when it was shown that lymphodepletion using a nonmyeloablative chemotherapy regimen administered immediately before TIL transfer could lead to increased cancer regression, as well as the persistent oligoclonal repopulation of the host with the transferred antitumor lymphocytes (13). In some patients, the administered anti-tumor cells represented up to $80 \%$ of the $\mathrm{CD}^{+} \mathrm{T}$ cells in the circulation months after the infusion. 
Lymphocyte cultures can be grown from many tumor histologies; however, melanoma appeared to be the only cancer that reproducibly gave rise to TIL cultures capable of specific antitumor recognition. The stimulus to more widely apply ACT to treat multiple human cancers led to studies of the genetic engineering of lymphocytes to express antitumor receptors. Following mouse models (14), it was shown for the first time in humans in 2006 that administration of normal circulating lymphocytes transduced with a retro-virus encoding a TCR that recognized the MART-1 melanoma-melanocyte antigen could mediate tumor regression (15). Administration of lymphocytes genetically engineered to express a chimeric antigen receptor (CAR) against the B cell antigen CD19 was shown in 2010 to mediate regression of an advanced B cell lymphoma (16). These findings of the use of either naturally occurring or genetically engineered antitumor $\mathrm{T}$ cells set the stage for the extended development of ACT for the treatment of human cancer.

\section{ACT using TILs is an effective immunotherapy for patients with metastatic melanoma}

Adoptive cell therapy using autologous TILs is the most effective approach to induce complete durable regressions in patients with metastatic melanoma (Table 1). The general approach for growing and administering human TILs is shown in Fig. 1. The resected melanoma specimen is digested into a single-cell suspension or divided into multiple tumor fragments that are individually grown in IL-2. Lymphocytes overgrow, destroy tumors within 2 to 3 weeks, and give rise to pure cultures of lymphocytes that can be tested for reactivity against tumors, if available, in co-culture assays. Individual cultures are then rapidly expanded in the presence of excess irradiated feeder lymphocytes, an antibody targeting the epsilon subunit within the human CD3 complex of the TCR, and IL-2. By 5 to 6 weeks after resecting the tumor, up to $10^{11}$ lymphocytes can be obtained for infusion into patients. A substantial increase in cell persistence and the incidence and duration of clinical responses was seen when patients received a lymphodepleting preparative regimen before the cell infusion (13). It might be possible to optimize the intensity or duration of the lymphodepletion that is employed, but the most frequently used lymphodepleting preparative regimen consists of $60 \mathrm{mg} / \mathrm{kg}$ cyclophosphamide for 2 days and $25 \mathrm{mg} / \mathrm{m}^{2}$ fludarabine adminis tered for 5 days followed by cells and IL-2 given at 720,000 IU/kg to tolerance (Fig. 2). In a pilot study in the Surgery Branch, National Cancer Institute (NCI), objective cancer regressions by RECIST criteria (Response Evaluation Criteria in Solid Tumors) were seen in 21 of 43 patients (49\%), including 5 patients (12\%) who underwent complete cancer regression (13). When 200 or 1200 centigray (cGy; $1 \mathrm{~Gy}=100$ rads) totalbody irradiation (TBI) was added to the preparative regimen in pilot trials of 25 patients each, objective response (OR) rates 34 complete responders thus far seen in the two trials at the NCI, only one has recurred, and only one patient with complete regression received more than one treatment. The brain is not a sanctuary site, and regression of brain metastases has been observed (21). Prior treatment with targeted therapy using the Braf inhibitor vemurafenib (Zelboraf) does not appear to affect the likelihood of having an OR to ACT treatment in patients with melanoma. ACT can also be effective after other immunotherapies have failed. Of the 194 patients treated of 52 and $72 \%$ were seen, including 20 and $40 \%$ complete regressions. However, there were no statistically significant differences in the OR 
rates between preparative regimens $(13,17)$. Twenty of the 93 patients $(22 \%)$ in these trials had complete regressions, and 19 (20\%) have not experienced recurrences at follow-up times of 5 to 10 years and are probably cured. A prospective randomized study comparing the chemotherapy preparative regimen alone versus chemotherapy plus the addition of $1200 \mathrm{cGy}$ TBI in 101 patients was recently concluded at the NCI, National Institutes of Health (NIH), and results are pending.

In the combined experience of the treatment of 194 patients using TILs grown from individual melanoma fragments at the NCI (Bethesda, Maryland), 107 patients $(55 \%)$ have shown ORs. Similar OR rates to TIL therapy have been reported by multiple groups, including those from the Moffitt Cancer Center (Tampa, Florida) (38\% OR rate) (18), the MD Anderson Cancer Center (Houston, Texas) (48\% OR rate) (19), and the Ella Cancer Institute (Ramat Gan, Israel) (40\% OR rate) (20) (Table 1).

There is no relation between the bulk of disease or the site of metastases and the likelihood of achieving a complete cancer regression (17). Of the in the NCI trials, OR rates in patients who had no prior therapy or who progressed through IL-2, antibody to cytotoxic T lymphocyte-associated protein 4 (anti-CTLA-4), anti-PD1, or Braf inhibitors were 48, 63, 42,50 , and $43 \%$, respectively.

Lymphodepletion appears to be an important component of ACT, and mouse models have shown that lymphodepletion given before cell transfer can increase the effectiveness of treatment more than 10-fold. In the clinic, the persistence of T cells was once a rarity (22), but in trials conducted after the initiation of lymphodepleting therapy, adoptively transferred $\mathrm{T}$ cells could comprise the majority of the peripheral blood $\mathrm{CD}^{+}$cells 1 month after transfer (13). The cellular basis of the effect of lymphodepletion is complex and still not completely understood. In mouse models, myeloid-derived suppressor cells and CD4 ${ }^{+}$FoxP3 regulatory $\mathrm{T}$ cells can be found at high levels in tumors in vivo and can depress immune responses in the mouse tumor microenvironment (23). In accord with these preclinical findings, preparative chemotherapy in humans severely depletes lymphocytes and myeloid cells from the circulation at the time of cell infusion, although the rate of reappearance of FoxP3 inhibitory $\mathrm{T}$ cells after lymphodepletion was inversely correlated with clinical response (24). Levels of homeostatic cytokines, which promote $\mathrm{T}$ cell proliferation and survival, are dramatically induced upon lymphodepletion (25) in mouse models. In humans, lymphodepletion leads to the appearance in the circulation of the T cell growth factor IL-15, which serves to promote the expansion of the transferred cells in the absence of competing endogenous lymphocytes (26). Further, lympho-depletion can enhance the translocation of commensal microflora across mucosal barriers in the mouse, and this can enhance the effect of ACT by stimulating Toll-like receptors (27) to activate antigen-presenting cells (APCs). These preclinical results have highly affected clinical translation, and it seems likely that immune ablation will be a part of future cell-based treatments in patients with cancer.

Adoptive cell therapy is a "living" treatment, and administered lymphocytes can expand more than 1000-fold after administration. Studies in mouse models, including those involving the injection of human cells into immunodeficient animals, have emphasized the importance of the differentiation state of the infused cells $(28,29)$. The phenotypic and 
functional status of less differentiated murine cells is highly positively correlated with their ability to eliminate vascularized tumor in vivo. These findings are in accordance with the high positive correlation between the persistence of the transferred TILs in the circulation of patients at 1 month and with the induction of partial and complete clinical responses (17). Further, one clinical study showed a strong correlation between expression of the phenotypic marker CD27, which is associated with cells early in their differentiation pathway, and clinical response (17). The presence of longer telomeres as a correlate of clinical response was seen in one study (17) but not in another (18).

The observation that melanoma TILs can mediate durable, complete, and probably curative cancer regression in patients with metastatic melanoma has raised considerable interest in the possible use of TILs for the treatment of multiple cancer types. Although TILs can be grown in vitro from virtually all tumors, only melanomas consistently give rise to TILs with antitumor reactivity. In an attempt to gain insight into the possible extension of ACT to the treatment of other common cancers, extensive studies of the antigens recognized by TILs have been pursued.

\section{Melanoma TILs recognize the products of cancer mutations}

Early studies identified two nonmutated melanomamelanocyte differentiation proteins, MART-1 and gp100, that were often recognized by melanoma TILs $(30,31)$. Melanocytes in the skin, eye, and ear express the MART-1 and gp100 proteins, and yet toxicity targeting these proteins was not seen in the majority of patients treated with TILs who underwent complete cancer regression. In contrast, when a high-affinity TCR against MART-1 or gp100 was inserted into lymphocytes used for ACT, profound eye and ear toxicity was often seen in the absence of antitumor activity, which suggests that the reactivity against melanomamelanocyte antigens was not the decisive target resulting in the in vivo antitumor activity of melanoma TILs (32).

A study of exomic mutation rates in more than 3000 tumor-normal pairs revealed that the frequency of nonsynonymous mutations varied more than 1000-fold across different cancer types (33). Pediatric cancers exhibited mutation frequencies as low as $0.1 / \mathrm{Mb}$, whereas melanomas and lung cancers often exceeded 100 mutations/Mb. The suggestion that mutations might be targets of immune recognition of tumor cells has been around for some time (34). The responsiveness of melanoma to a variety of immunotherapy approaches such as ACT, IL-2, anti-CTLA-4, and anti-PD-1 suggested that peptide epitopes encoded by the large number of mutations in melanoma might be the targets of TIL therapy (35). Support for this hypothesis comes from recent observations that anti-PD-1 can mediate ORs not only in patients with melanoma but also in patients with lung and bladder cancer, the two tumor types closest to melanoma with a high frequency of mutations (36). A patient successfully treated with anti-CTLA-4 generated circulating $T$ cells that recognized a distinct mutation in the melanoma (37). Another study suggested that increased numbers of exomic mutations in a cancer correlated with better outcomes (38).

New approaches using whole-exomic sequencing of tumor-normal pairs in patients with melanoma have consistently identified non-synonymous cancer mutations recognized by 
autologous TILs that mediated complete cancer regressions $(39,40)$. However, not all expressed mutations can be recognized by $\mathrm{T}$ cells. Proteins incorporating the mutations must be processed to short peptides of $\sim 9$ amino acids for major histocompatibility complex (MHC) class 1 and a bit longer for MHC class 2; these peptides are then presented on the cell surface. One approach to identify the immunogenic mutations that we have taken is to identify 21 - to 25 -amino acid polypeptides, each one containing a mutated amino acid flanked by 10 to 12 normal residues. Using peptide-MHC binding algorithms, these polypeptides can then be scanned to identify peptides with high binding to individual MHC molecules of the patient. The top-predicted binding peptides are then synthesized and tested for recognition by coculture with TILs that mediated cancer regression. This method depends on the accuracy of peptide-MHC binding algorithms, which are often inadequate for many of the less frequent MHC molecules (39).

An alternate method eliminates the need for predicted peptide binding to MHC and enables the screening of all candidate peptides on all MHC loci in a single test (40) (Fig. 3). As above, minigenes, rather than polypeptides, are constructed that encode each mutated amino acid flanked by 10 to 12 amino acids. Strings of 6 to 20 minigenes are then linked into tandem mini-genes, and these DNA constructs are subsequently cloned into an expression plasmid and in vitro transcribed to RNA, which is electroporated into the patient's autologous APCs. These APCs present all mutated peptides capable of being processed and binding to any of the patient's class 1 or class 2 MHC molecules. Culture of the patient's TILs with these APCs can identify the tandem minigene as well as the individual minigene responsible for tumor recognition. Using these approaches, TILs from 21 patients with mela noma that responded to ACT identified 45 mutations presented on a variety of class 1 and class 2 MHC molecules. Thus far, every mutation recognized by TILs was distinct (i.e., each from a different expressed protein), with none shared by another melanoma in the set studied. These findings provide suggestive evidence that melanoma TILs capable of mediating antitumor responses were recognizing random somatic mutations in the cancer. In many cases, multiple mutations were recognized by an individual TIL population. The concept that cancer regressions after immunotherapy are the result of targeting mutations explains why patients can experience tumor regression without auto-immune sequelae. Conversely, the ineffectiveness of the vast number of therapeutic cancer vaccines that targeted nonmutated self-proteins can also be explained $(41,42)$. Whereas strong reactivity to self-antigens causes autoimmune toxicity, vaccines against self-antigens trigger the expansion of low-affinity TCRs against self-proteins that escaped negative selection in the thymus. This raises the possibility that vaccines targeting mutated immunogenic epitopes may be much more effective. The specific targeting of individual mutated antigens in a patient's cancer presents a daunting problem for widespread therapeutic application of ACT but also presents an opportunity to develop treatments for multiple cancer types. Schumacher and Schreiber discuss additional aspects for targeting mutated antigens in this issue (43).

\section{TILs from common epithelial cancers can also recognize cancer mutations}

A recent report has shown that the mutated antigens in a nonmelanoma epithelial cancer can give rise to immune responses, despite the low number of mutations in these cancers (44). 
Exomic sequencing of a metastatic cholangio-carcinoma in a patient who had progressed through multiple chemotherapies revealed 26 nonsynonymous mutations. Tandem minigenes that encoded each mutated amino acid and its flanking sequences were constructed and electroporated into the patient's APCs. CD4 cells from TIL cultures from this patient's tumor recognized the ERBB2IP mutation restricted by the MHC class 2 antigen HLA-DQ O6. ERBB2IP is a tumor suppressor that binds to ERBB2 and attenuates downstream RAS/ERK signaling. Despite the lack of an objective clinical response to the administration of bulk autologous TILs in this patient, administration of TILs that were selected to contain more than 95\% ERBB2IP mutation-reactive TILs mediated a dramatic regression of liver and lung metastases ongoing beyond 1 year. This result provides compelling evidence that mutation-reactive $\mathrm{T}$ cells are capable of mediating in vivo tumor regression in patients with this epithelial cancer. Further, the findings suggest that this treatment approach may be suitable for patients with other common epithelial cancers that are not normally considered to be immunogenic.

Mutations that are targeted may be driver mutations essential for the malignant phenotype of the cell, or alternatively, the TILs may contain reactivity against multiple immunogenic passenger mutations, which would decrease the likelihood that the loss of any individual antigen would subvert the clinical antitumor response. TIL populations can be highly polyclonal and thus are likely to be capable of potentially recognizing multiple antigens simultaneously. Given their curative potential, it seems likely that TILs are able to recognize antigens expressed by cancer stem cells. Although some of the mutations are probably driver mutations because they are found in expressed genes associated with known oncogenic pathways (e.g., mutated $\beta$-catenin), many of the targets of TILs may well be passenger mutations.

\section{Genetic engineering of lymphocytes for use in ACT}

In an attempt to broaden the reach of ACT to other cancers, techniques were developed to introduce antitumor receptors into normal T cells that could be used for therapy (Fig. 4). The specificity of $\mathrm{T}$ cells can be redirected by the integration of genes encoding either conventional alpha-beta TCRs or CARs. CARs were pioneered by Gross and colleagues in the late 1980s (45) and can be constructed by linking the variable regions of the antibody heavy and light chains to intracellular signaling chains such as CD3-zeta, often in cluding costimulatory domains encoding CD28 (46) or CD137 to fully activate T cells $(47,48)$. CARs can provide non-MHC-restricted recognition of cell surface components and can be introduced into $\mathrm{T}$ cells with high efficiency using viral vectors.

An important question confronting the use of genetically engineered cells in the ACT of cancer involves selection of the ideal human $\mathrm{T}$ cell sub-population into which the gene should be introduced, as well as the selection of appropriate antigenic targets of the introduced TCRs or CARs. Preclinical studies in mouse models strongly suggest that improved antitumor responses are seen when $\mathrm{T}$ cells in early stages of differentiation (such as naïve or central memory cells) are transduced (49), a result supported by studies in monkeys showing improved in vivo persistence of infused central memory compared with effector memory cells (50). CD8 ${ }^{+} \mathrm{T}$ cells can be categorized into distinct memory subsets 
based on their differentiation states. We and others have found that $\mathrm{CD} 8^{+} \mathrm{T}$ cells follow a progressive pathway of differentiation from naïve $\mathrm{T}$ cells into central memory and effector memory $\mathrm{T}$ cell populations [summarized in (51)]. $\mathrm{CD}^{+} \mathrm{T}$ cells paradoxically lose antitumor $\mathrm{T}$ cell functionality as they acquire the ability to lyse target cells and to produce the cytokine interferon- $\gamma$, qualities thought to be important in their antitumor efficacy (52). The differentiation state of $\mathrm{CD}^{+} \mathrm{T}$ cells is inversely related to their capacity to proliferate and persist (52-54). These findings may be clinically relevant, and younger $\mathrm{T}$ cells are statistically positively correlated with clinical effectiveness in ACT trials (17). It seems clear that, like many organ systems in the body, $\mathrm{CD}^{+} \mathrm{T}$ cells can exist in a stem cell-like state, capable of clonal repopulation. Human T memory stem cells express a gene program that enables them to proliferate extensively and can further differentiate into other $\mathrm{T}$ cell populations (29).

Much of the existing work in cancer immunotherapy has focused on $\mathrm{CD}^{+} \mathrm{T}$ cells. However, $\mathrm{CD}^{+} \mathrm{T}$ cells can also efficiently promote tumor rejection. $\mathrm{CD}^{+} \mathrm{T}$ cells do not merely enhance $\mathrm{CD}^{+} \mathrm{T}$ cell function, but they also play a more direct role in tumor elimination. This notion has been validated recently in humans (44). The roles that $\mathrm{CD}^{+} \mathrm{T}$ cells play in the antitumor immune response crucially depend on their polarization, which is determined by their expression of key transcription factors. $\mathrm{CD}^{+}$cells can destroy tumor cells, and recent evidence suggests that adoptively transferred $\mathrm{T}$ helper 17 cells can promote long-lived antitumor immunity (55).

\section{Toxicity of ACT when targeting antigens shared by tumors and normal tissue}

The marked potency of $\mathrm{T}$ cells enables the recognition of minute levels of antigen expressed on normal cells. Thus, targeting normal, nonmutated antigenic targets that are expressed on normal tissues but overexpressed on tumors has led to severe on-target, off-tumor toxicity in patients. Suitable antigens to target are those presented exclusively on the cancer or, alternatively, on normal cells that are not essential for survival.

The first successful application of ACT using genetically engineered lymphocytes treated 17 patients with metastatic melanoma using autologous $\mathrm{T}$ cells transduced with a weakly avid human TCR recognizing the MART-1 melanoma-melanocyte differentiation antigen (15). Two patients experienced objective partial regressions of metastatic melanoma, and in both patients the transferred cells could be found in the peripheral blood 1 year after cell infusion. This approach was expanded to 36 patients with metastatic melanoma who received highavidity TCRs that recognized either the MART-1 or gp100 melanoma-melanocyte antigens (32). Although objective cancer regressions were observed in 30 and $19 \%$ of patients who received the MART-1 or gp100 TCR, respectively, severe off-tumor, on-target toxicity was seen in the skin, eyes, and ears of patients due to the expression of melanocytes in these organs. These findings coincided with severe eye toxicity seen in mice when targeting melanocyte antigens and provided an early demonstration of the power of $\mathrm{T}$ cell therapy (56). The treatment of patients with renal cancer using T cells encoding a CAR against carbonic anhydrase 9, which is overex-pressed in renal cancer, led to severe liver toxicity 
due to expression of this antigen in biliary duct epithelium (57). A high-affinity TCR against the carcinoembryonic antigen was used to treat patients with metastatic colorectal cancer that expressed high levels of this antigen (58). All three patients experienced life-threatening coli tis and colonic hemorrhage that precluded further use of this TCR, even though one patient exhibited a partial response of liver metastases. Unexpected toxicities can also result when previously unknown cross-reactivities are seen that target normal self-proteins expressed in vital organs. MAGE-A3, a cancer-testes antigen to be discussed in more detail below, is not known to be expressed in any normal tissues. However, targeting an HLAA*0201-restricted peptide in MAGE-A3 caused severe damage to gray matter in the brain, resulting in two deaths because this TCR recognized a different but related epitope expressed by MAGE-A12, expressed at very low levels in the brain (59). It should also be noted that CARs are capable of toxicity against self-antigens as well. Acute pulmonary toxicity resulting in death was observed after infusion of CAR T cells specific for ERBB2, which seemed likely due to the recognition of low levels of this antigen on pulmonary epithelium (60).

Several groups have attempted to affinity-enhance TCRs by altering amino acids in the antigen-combining sites of the TCR $(61,62)$. By removing the protective effects of negative thymic selection that eliminate high-affinity TCRs against normal proteins, these modified TCRs could potentially recognize new and unrelated determinants. Two patients (one with multiple myeloma and one with melanoma) were treated with an HLA-A1-restricted MAGE-A3-specific TCR whose affinity was enhanced by this site-specific mutagenesis, and both experienced fatal cardiogenic shock due to the recognition of an HLA-A1-restricted peptide derived from an unrelated protein, titin, present in cardiac muscle (63). Thus, methods aimed at enhancing the affinities of TCRs can be fraught with problems of unexpected toxicities, which remain difficult to predict. Of course, the same pitfalls of unexpected toxicities may apply to the use of novel CARs.

\section{Targeting antigens expressed on cancers and nonessential human tissues}

Cancers that express target molecules shared with nonessential normal organs represent potential targets for human cancer immunotherapy using ACT. A prominent example of such an antigen is the CD19 molecule expressed on more than $90 \%$ of B cell malignancies and on $\mathrm{B}$ cells at all stages of differentiation, excluding plasma cells. Following preclinical work by many groups [summarized in (64-67)], the first successful clinical application of anti-CD19 CAR gene therapy in humans was reported in 2010 (16). Administration of autologous cells expressing the anti-CD19 CAR to a patient with refractory lymphoma resulted in cancer regression in a patient who remains progression-free after two cycles of treatment ongoing 4 years after treatment. Multiple groups have now shown the effectiveness of ACT targeting CD19 in patients with follicular lymphoma, large-cell lymphomas, chronic lymphocytic leukemia, and acute lymphocytic leukemia (68-72). On-target toxicity against CD19 results in B cell loss in the circulation and in the bone marrow and can be overcome by the periodic administration of immunoglobulin infusions. Sub stantial toxicity can be seen by the excessive release of cytokines by CAR-expressing cells, and thus, careful selection of the lymphodepleting preparative regimen and the cell dose is required to safely apply ACT targeting CD19, as well as many other antigens now under experimental study (72). 
Dramatic regressions of lymphomas and leukemias with ACT have elicited considerable enthusiasm, although most reports contain fewer than 20 patients, and fewer than 200 patients have been treated worldwide. The introduction of CARs into lymphocytes has mainly used gammaretroviruses and lentiviruses, although nonviral approaches such as transposon-transposase systems (73) and CRISPR-cas (CRISPR, clustered regularly interspaced palindromic repeat) technology to introduce genes are also being explored (74). The single-chain antibody governs recognition of the antigen to be targeted, although the $\mathrm{T}$ cell is activated via the CD3-zeta chain signaling domain. In addition to the zeta chain, a variety of costimulatory molecules have been employed in retroviral constructs such as CD27, CD28, CD134, CD137, or ICOS that can profoundly influence the function of the CAR [reviewed in (64-66)]. Optimization of these costimulatory domains is a subject of active study. The results of CAR therapy for B cell malignancies might be confounded by the sensitivity of lymphomas and leukemias to the preparative chemotherapy regimen. Thus, delineation between the effects of the preparative therapy and those of the CAR T cells needs to be considered.

Multiple other B cell antigens are being studied as targets, including CD22, CD23, ROR-1, and the immunoglobulin light-chain idiotype expressed by the individual cancer (65). CARs targeting either CD33 or CD123 have been studied as a therapy for patients with acute myeloid leukemia, though the expression of these molecules on normal precursors can lead to prolonged myeloablation (75). BCMA is a tumor necrosis factor receptor family protein expressed on mature B cells and plasma cells and can be targeted on multiple myeloma (65). The Reed-Sternberg cell expresses CD30, and this target is being explored as a treatment for patients with refractory Hodgkin lymphoma (75-77).

Although CARs are being successfully applied to the treatment of hematologic malignancies, the lack of shared antigens on the surface of solid tumors that are not also expressed on essential normal tissues has severely limited the application of CARs to the treatment of solid tumors. Thyroglobulin is a potential target for some patients with thyroid cancers because thyroglobulin is present only in the thyroid gland and not on solid tissues. Neuroblastomas express GD2, which has been targeted by CARs (78). Mesothelin has also been forwarded as a potential target, although it is also expressed on normal tissues, including cells in the pericardium and pleural and pertitoneal linings (79). A search is ongoing for other tissue-specific surface antigens expressed on tissues that are not essential for survival.

Cancer-testis antigens are a family of intracellular proteins that are expressed during fetal development but have highly restricted expression in adult normal tissues (80). There are more than 100 different members of this family of molecules whose expression is epigenetically up-regulated from 10 to $80 \%$ of cancer types using highly sensitive techniques. However, initial enthusiasm for targeting cancer-testes antigens has been tempered by the lack of high levels of protein expression of these antigens. Approximately $10 \%$ of common cancers appear to express enough protein to be suitable targets for antitumor $\mathrm{T}$ cells. There are low levels of some cancer-testes antigens expressed on normal tissues, and this can lead to untoward toxicities. The NYESO-1 cancer-testes antigen has been targeted via a human TCR transduced into autologous cells (81). ORs were seen in 5 of 
11 patients with metastatic melanoma and 4 of 6 patients with highly refractory synovial cell sarcoma.

\section{Looking to the future of ACT for the treatment of cancer}

The continued development of ACT, as well as other immunologic approaches to the treatment of cancer, depends on the identification of suitable targets for immunologic attack. Although CARs have been successful in the treatment of hematologic malignancies and are likely to soon join the mainstream of oncologic treatment, the ability to treat common epithelial solid cancers, which account for $\sim 90 \%$ of all cancer fatalities, is severely limited by the lack of suitable targets exclusive to cancer. Extensive searches for monoclonal antibodies that can recognize distinct determinants on the surface of solid cancers but not normal tissues have been in progress for more than 30 years, but few suitable determinants have been found. The EGFRvIII mutation on $\sim 40 \%$ of high-grade glioblastomas is a rare example of a shared-surface mutation, and attempts to target this molecule using CARs are in progress (82). Shared mutations in intracellular proteins involved in oncogenesis—such as Braf in melanomas and Kras in pancreatic and other solid cancers-would be ideal ACT targets using conventional alpha-beta TCRs, though immunogenic epitopes have not yet been identified in these molecules. Driver and random somatic mutations occurring in many solid cancers may represent excellent targets for the treatment of solid tumors.

Opportunities to improve ACT involve the identification and development of specific antitumor T cells with the functional properties optimal for tumor destruction (83). One approach under active evaluation is the growth of cells under conditions that enable in vitro proliferation while limiting differentiation, such as the use of IL-21 or inhibitors that target the kinase AKT $(84,85)$. Improved specific lymphodepleting preparative regimens and better design of the transducing vectors, including the incorporation of optimal costimulatory molecules, are likely to improve clinical results. Introduction of genes encoding other molecules such as the cytokine IL-12, which can profoundly alter the tumor microenvironment to favor antitumor immunity, has shown substantial promise in animal models (86). Enhanced methods for regulating the expression of these highly potent cytokine genes would be an important part of incorporating them into clinical treatment. The incorporation of "suicide" genes that can enable destruction of the transferred cells could add an extra level of safety when exploring genetic changes in lymphocytes (87).

Adoptive cell therapy is a more complex approach to the delivery of cancer treatment than many other types of immunotherapy and has often been criticized as impractical and too costly for widespread application. The need to develop highly personalized treatments for each patient does not fit into the paradigm of major pharmaceutical companies that depend on "off-the-shelf" reagents that can be widely distributed. However, curative immunotherapies for patients with common epithelial cancers will probably dictate the need for more personalized approaches. Several new biotechnology companies have arisen to meet the need to expand a patient's lymphocytes, and detailed genetic analysis of individual tumors is already commonplace at large academically affiliated medical centers. Although multiple commercial models have been proposed, widespread application of ACT will probably depend on the development of centralized facilities for producing tumor-reactive 
TILs or genetically modified lymphocytes that can then be delivered to the treating institution. The effectiveness of treatment will need to trump convenience of administration in the application of new effective approaches to cancer immunotherapy.

\section{ACKNOWLEDGMENTS}

We thank the Center for Cancer Research, NCI, NIH, Bethesda, Maryland, and the Milstein Family Foundation for their generous support.

\section{REFERENCES AND NOTES}

1. Delorme EJ, Alexander P, Lancet 284, 117-120 (1964).

2. Fefer A, Cancer Res. 29, 2177-2183 (1969). [PubMed: 5369675]

3. Fernandez-Cruz E, Woda BA, Feldman JD, J. Exp. Med 152, 823-841 (1980). [PubMed: 6968337]

4. Berendt MJ, North RJ, J. Exp. Med 151, 69-80 (1980). [PubMed: 6444236]

5. Morgan DA, Ruscetti FW, Gallo R, Science 193, 1007-1008 (1976). [PubMed: 181845]

6. Rosenberg SA, Mulé JJ, Spiess PJ, Reichert CM,Schwarz SL, J. Exp. Med 161, 1169-1188 (1985). [PubMed: 3886826]

7. Eberlein TJ, Rosenstein M, Rosenberg SA, J. Exp. Med 156, 385-397 (1982). [PubMed: 6980254]

8. Donohue JH et al., J. Immunol 132, 2123-2128 (1984). [PubMed: 6607956]

9. Rosenberg SA et al., N. Engl. J. Med 313, 1485-1492 (1985). [PubMed: 3903508]

10. Rosenberg SA, Spiess P, Lafreniere R, Science 233, 1318-1321 (1986). [PubMed: 3489291]

11. Muul LM, Spiess PJ, Director EP, Rosenberg SA,J. Immunol 138, 989-995 (1987). [PubMed: 3100623]

12. Rosenberg SA et al., N. Engl. J. Med 319, 1676-1680 (1988). [PubMed: 3264384]

13. Dudley ME et al., Science 298, 850-854 (2002). [PubMed: 12242449]

14. Kessels HWHG, Wolkers MC, van den Boom MD,van den Valk MA, Schumacher TNM, Nat. Immunol 2, 957-961 (2001). [PubMed: 11577349]

15. Morgan RA et al., Science 314, 126-129 (2006). [PubMed: 16946036]

16. Kochenderfer JN et al., Blood 116, 4099-4102 (2010). [PubMed: 20668228]

17. Rosenberg SA et al., Clin. Cancer Res. 17, 4550-4557 (2011). [PubMed: 21498393]

18. Pilon-Thomas S et al., J. Immunother 35, 615-620 (2012). [PubMed: 22996367]

19. Radvanyi LG et al., Clin. Cancer Res. 18, 6758-6770 (2012). [PubMed: 23032743]

20. Besser MJ et al., Clin. Cancer Res. 19, 4792-4800 (2013). [PubMed: 23690483]

21. Hong JJ et al., Clin. Cancer Res. 16, $4892-4898$ (2010). [PubMed: 20719934]

22. Rosenberg SA et al., N. Engl. J. Med 323, 570-578 (1990). [PubMed: 2381442]

23. Bronte V et al., J. Immunol 161, 5313-5320 (1998). [PubMed: 9820504]

24. Yao X et al., Blood 119, 5688-5696 (2012). [PubMed: 22555974]

25. Gattinoni L et al., J. Exp. Med 202, 907-912 (2005). [PubMed: 16203864]

26. Dudley ME et al., J. Clin. Oncol 26, 5233-5239 (2008). [PubMed: 18809613]

27. Paulos CM et al., J. Clin. Invest 117, 2197-2204 (2007). [PubMed: 17657310]

28. Restifo NP, Dudley ME, Rosenberg SA, Nat. Rev. Immunol 12, 269-281 (2012). [PubMed: 22437939]

29. Gattinoni L et al., Nat. Med 17, 1290-1297 (2011). [PubMed: 21926977]

30. Kawakami Y et al., Proc. Natl. Acad. Sci. U.S.A. 91, 6458-6462 (1994). [PubMed: 8022805]

31. Kawakami Y et al., Proc. Natl. Acad. Sci. U.S.A. 91, 3515-3519 (1994). [PubMed: 8170938]

32. Johnson LA et al., Blood 114, 535-546 (2009). [PubMed: 19451549]

33. Lawrence MS et al., Nature 499, 214-218 (2013). [PubMed: 23770567]

34. Wölfel T et al., Science 269, 1281-1284 (1995). [PubMed: 7652577]

35. Gubin MM et al., Nature 515, 577-581 (2014). [PubMed: 25428507] 
36. Topalian SL et al., N. Engl. J. Med 366, 2443-2454 (2012). [PubMed: 22658127]

37. van Rooij RN et al., J. Clin. Oncol 31, e439-e442 (2013). [PubMed: 24043743]

38. Brown SD et al., Genome Res. 24, 743-750 (2014). [PubMed: 24782321]

39. Robbins PF et al., Nat. Med 19, 747-752 (2013). [PubMed: 23644516]

40. Lu YC et al., Clin. Cancer Res. 20, 3401-3410 (2014). [PubMed: 24987109]

41. Rosenberg SA, Yang JC, Restifo NP, Nat. Med 10, 909-915 (2004). [PubMed: 15340416]

42. Klebanoff CA, Acquavella N, Yu Z, Restifo NP, Immunol. Rev 239, 27-44 (2011). [PubMed: 21198663]

43. Schumacher TN, Schreiber RD, Science 348, 69-74 (2015). [PubMed: 25838375]

44. Tran E et al., Science 344, 641-645 (2014). [PubMed: 24812403]

45. Gross G, Waks T, Eshhar Z, Proc. Natl. Acad. Sci. U.S.A. 86, 10024-10028 (1989). [PubMed: 2513569]

46. Maher J, Brentjens RJ, Gunset G, Rivière I, Sadelain M, Nat. Biotechnol 20, 70-75 (2002).

[PubMed: 11753365]

47. Imai C et al., Leukemia 18, 676-684 (2004). [PubMed: 14961035]

48. Song DG et al., Cancer Res. 71, 4617-4627 (2011). [PubMed: 21546571]

49. Klebanoff CA et al., Proc. Natl. Acad. Sci. U.S.A. 102, 9571-9576 (2005). [PubMed: 15980149]

50. Berger C et al., J. Clin. Invest 118, 294-305 (2008). [PubMed: 18060041]

51. Gattinoni L, Powell DJ, Jr., Rosenberg SA, Restifo NP, Nat. Rev. Immunol 6, 383-393 (2006).

[PubMed: 16622476]

52. Gattinoni L et al., J. Clin. Invest 115, 1616-1626 (2005). [PubMed: 15931392]

53. Buchholz VR et al., Science 340, 630-635 (2013). [PubMed: 23493420]

54. Gerlach C et al., Science 340, 635-639 (2013). [PubMed: 23493421]

55. Muranski P et al., Immunity 35, 972-985 (2011). [PubMed: 22177921]

56. Palmer DC et al., Proc. Natl. Acad. Sci. U.S.A. 105, $8061-8066$ (2008). [PubMed: 18523011]

57. Lamers CH et al., Mol. Ther 21, 904-912 (2013). [PubMed: 23423337]

58. Parkhurst MR et al., Mol. Ther 19, 620-626 (2011). [PubMed: 21157437]

59. Morgan RA et al., J. Immunother 36, 133-151 (2013). [PubMed: 23377668]

60. Morgan RA et al., Mol. Ther 18, 843-851 (2010). [PubMed: 20179677]

61. Li Y et al., Nat. Biotechnol 23, 349-354 (2005). [PubMed: 15723046]

62. Robbins PF et al., J. Immunol 180, 6116-6131 (2008). [PubMed: 18424733]

63. Linette GP et al., Blood 122, 863-871 (2013). [PubMed: 23770775]

64. Gardner RA, Jensen MC, Cancer J. 20, 107-111 (2014). [PubMed: 24667954]

65. Gill S, June CH, Immunol. Rev 263, 68-89 (2015). [PubMed: 25510272]

66. Sadelain M, Brentjens R, Rivière I, Cancer Discov. 3, 388-398 (2013). [PubMed: 23550147]

67. Kochenderfer JN, Rosenberg SA, Nat. Rev. Clin. Oncol 10, 267-276 (2013). [PubMed: 23546520]

68. Kalos M et al., Sci. Transl. Med 3, 95ra73 (2011).

69. Grupp SA et al., N. Engl. J. Med 368, 1509-1518 (2013). [PubMed: 23527958]

70. Brentjens RJ et al., Sci. Transl. Med 5, 177ra38 (2013).

71. Kochenderfer JN et al., J. Clin. Oncol 33, 540-549 (2015). [PubMed: 25154820]

72. Lee DW et al., Blood 124, 188-195 (2014). [PubMed: 24876563]

73. Singh H, Huls H, Kebriaei P, Cooper LJ, Immunol. Rev 257, 181-190 (2014). [PubMed: 24329797]

74. Doudna JA, Charpentier E, Science 346, 1258096 (2014). [PubMed: 25430774]

75. Pizzitola I et al., Leukemia 28, 1596-1605 (2014). [PubMed: 24504024]

76. Savoldo B et al., Blood 110, 2620-2630 (2007). [PubMed: 17507664]

77. Gopal AK et al., Blood 125, 1236-1243 (2015). [PubMed: 25533035]

78. Louis CU et al., Blood 118, 6050-6056 (2011). [PubMed: 21984804]

79. Zhao Y et al., Cancer Res. 70, 9053-9061 (2010). [PubMed: 20926399] 
80. Scanlan MJ, Gure AO, Jungbluth AA, Old LJ, Chen YT, Immunol. Rev 188, 22-32 (2002). [PubMed: 12445278]

81. Robbins PF et al., J. Clin. Oncol 29, 917-924 (2011). [PubMed: 21282551]

82. Morgan RA et al., Hum. Gene Ther. 23, 1043-1053 (2012). [PubMed: 22780919]

83. Gros A et al., J. Clin. Invest 124, 2246-2259 (2014). [PubMed: 24667641]

84. Li Y, Bleakley M, Yee C, J. Immunol 175, 2261-2269 (2005). [PubMed: 16081794]

85. Crompton JG et al., Cancer Res. 75, 296-305 (2015). [PubMed: 25432172]

86. Kerkar SP et al., J. Clin. Invest 121, 4746-4757 (2011). [PubMed: 22056381]

87. Di Stasi A et al., N. Engl. J. Med 365, 1673-1683 (2011). [PubMed: 22047558]

88. Rosenberg SA et al., J. Natl. Cancer Inst. 86, 1159-1166 (1994). [PubMed: 8028037]

89. Hinrichs CS. American Society of Clinical Oncology (ASCO) Annual Meeting, Chicago, IL, 30

May to 3 June 1014 (ASCO, Alexandria, VA, 2014); 2014. abstract LBA3008http://

meetinglibrary.asco.org/content/129263-144.

90. Hunder NN et al., N. Engl. J. Med 358, 2698-2703 (2008). [PubMed: 18565862]

91. Chapuis AG et al., Sci. Transl. Med 5, 174ra27 (2013).

92. Maude SL et al., N. Engl. J. Med 371, 1507-1517 (2014). [PubMed: 25317870]

93. Davila ML et al., Sci. Transl. Med 6, 224ra25 (2014).

94. Lee DW et al., Lancet 385, 517-528 (2015). [PubMed: 25319501] 


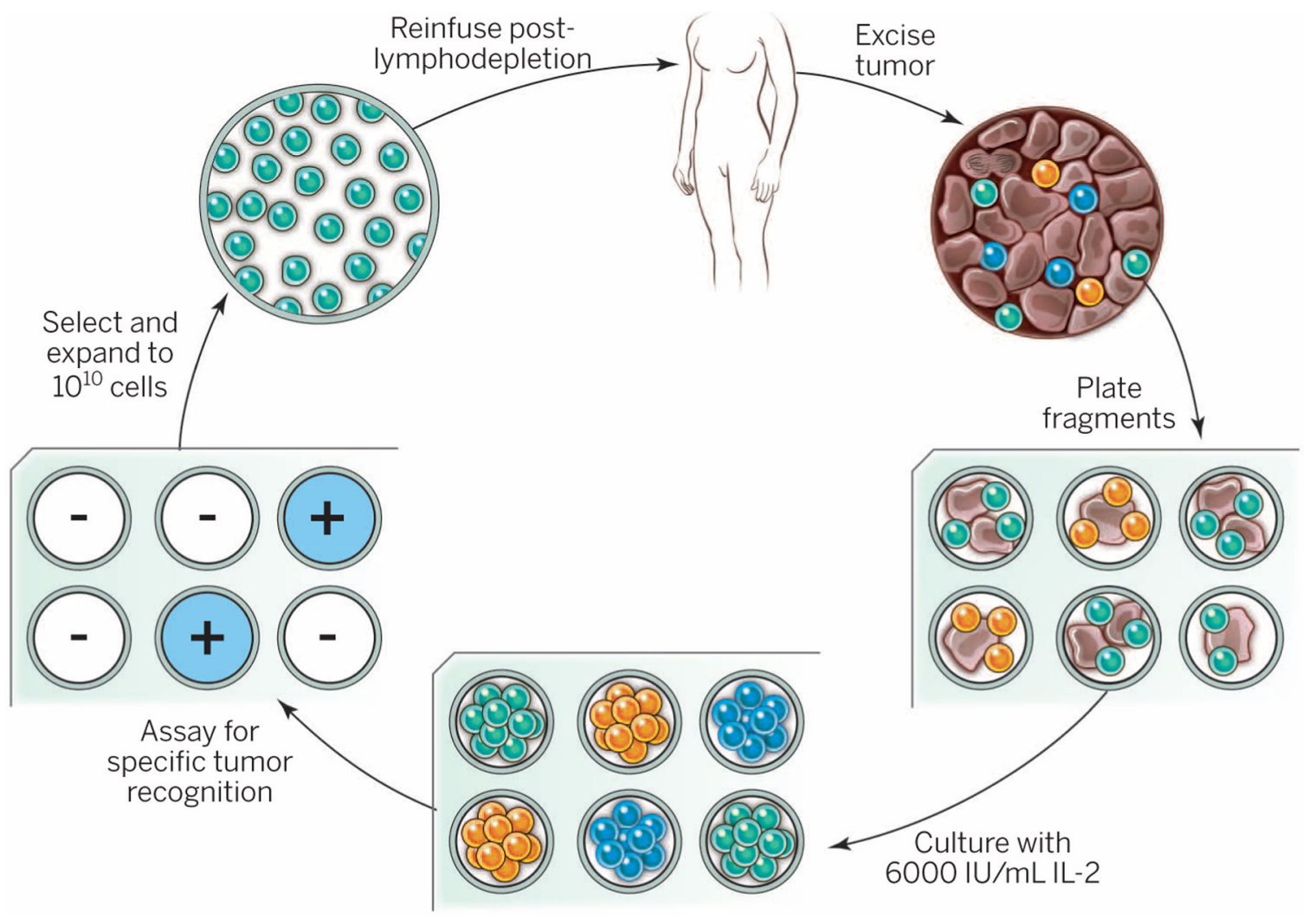

Fig. 1. General schema for using the adoptive cell transfer of naturally occurring autologous TILs.

The resected melanoma specimen is digested into a single-cell suspension or divided into multiple tumor fragments that are individually grown in IL-2. Lymphocytes overgrow, destroy tumors within 2 to 3 weeks, and generate pure cultures of lymphocytes that can be tested for reactivity in coculture assays. Individual cultures are then rapidly expanded in the presence of excess irradiated feeder lymphocytes, OKT3, and IL-2. By approximately 5 to 6 weeks after resecting the tumor, up to $10^{11}$ lymphocytes can be obtained for infusion into patients. 


\section{Lymphodepletion prior to T cell transfer is followed by immune reconstitution}

Peripheral blood cell count

6000 cells per $\mathrm{mm}^{3}$.

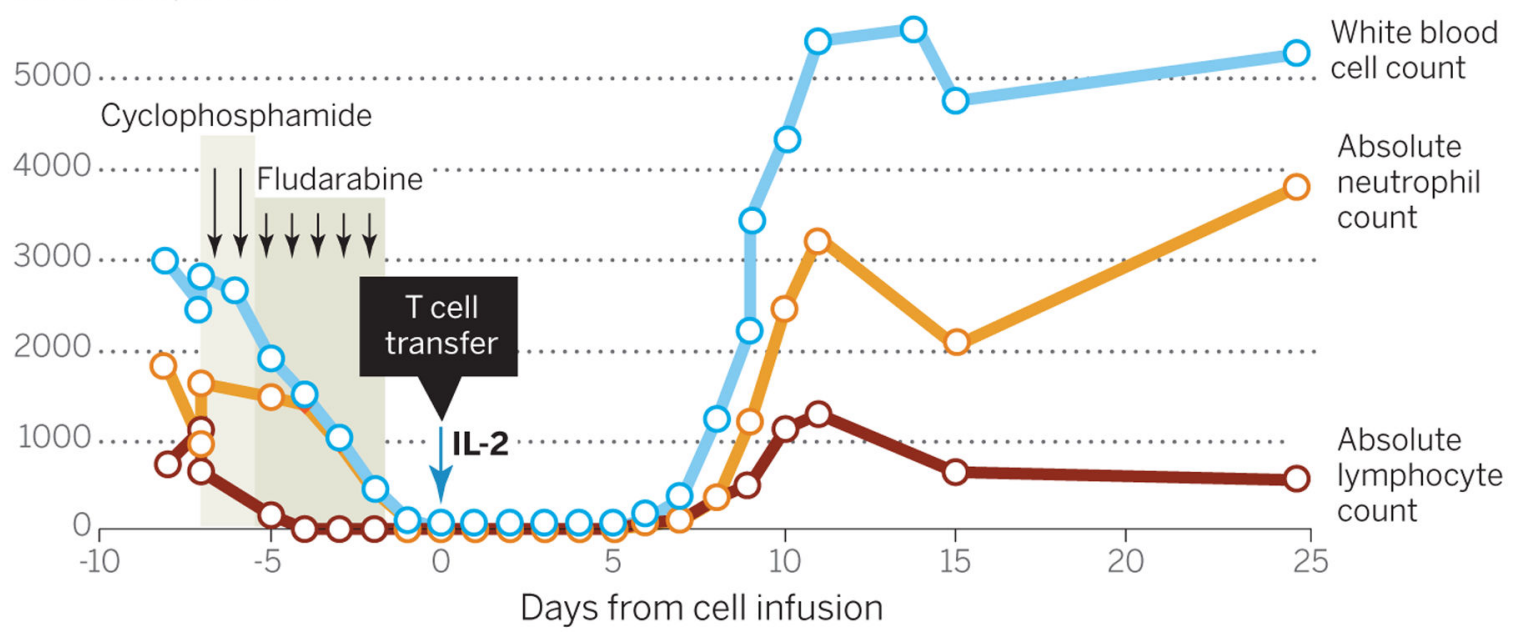

Fig. 2. A substantial increase in cell persistence and the incidence and duration of clinical responses is observed when patients received a lymphodepleting preparative regimen before the cell infusion.

The most frequently used lymphodepleting preparative regimen consists of $60 \mathrm{mg} / \mathrm{kg}$ cyclophosphamide given for 2 days and $25 \mathrm{mg} / \mathrm{m}^{2}$ fludarabine administered over 5 days, followed by $\mathrm{T}$ cells and IL-2 administration. 


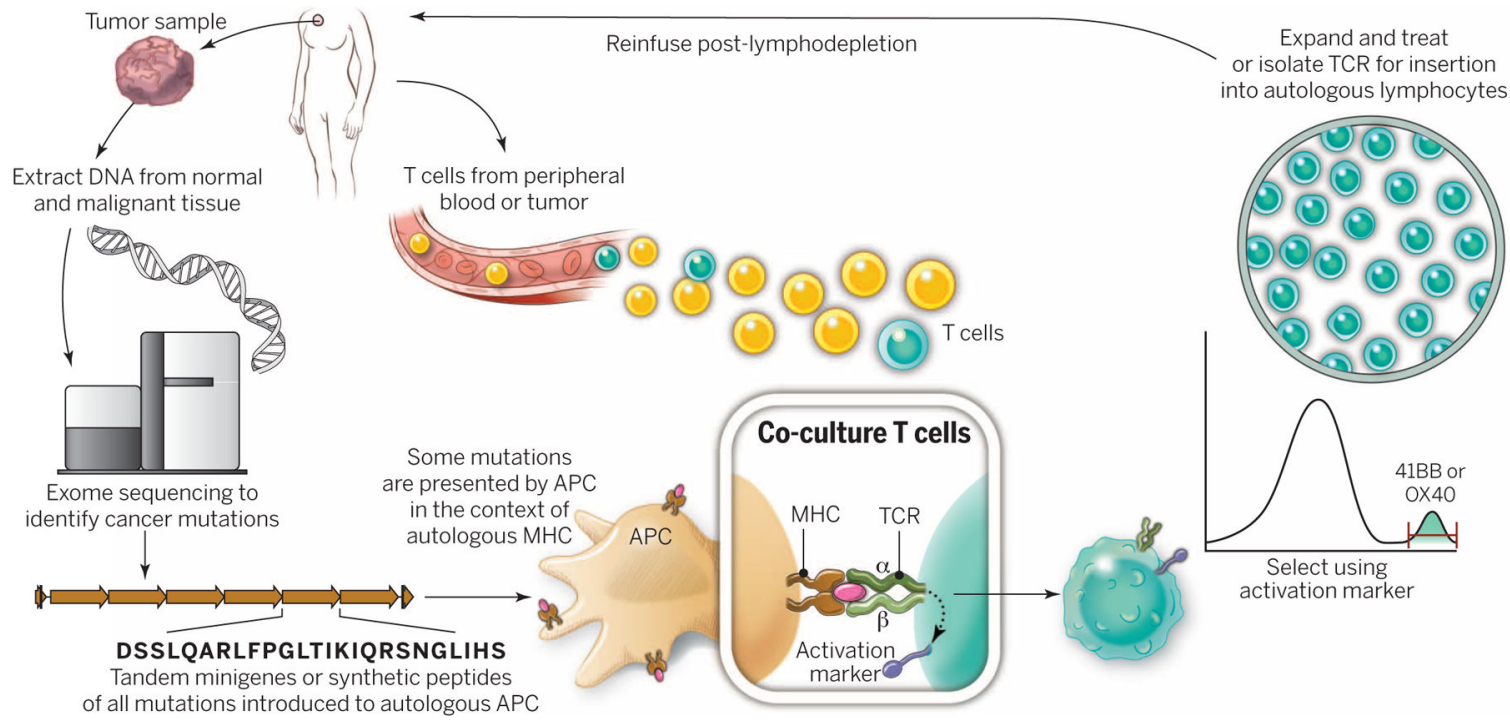

Fig. 3. A "blueprint" for the treatment of patients with $T$ cells recognizing tumor-specific mutations.

The sequences of exomic DNA from tumor cells and normal cells from the same patient are compared to identify tumor-specific mutations. Knowledge of these mutations can then be used to synthesize either minigenes or polypeptides encoding each mutated amino acid flanked by 10 to 12 amino acids. These peptides or minigenes can be expressed by a patient's autologous APCs, where they are processed and presented in the context of a patient's MHC. Coculture of the patient's T cells with these APCs can be used to identify all mutations processed and presented in the context of all of a patient's MHC class I and class II molecules. The identification of individual mutations responsible for tumor recognition is possible because $\mathrm{T}$ cells express activation markers, such as $41 \mathrm{BB}\left(\mathrm{CD} 8^{+} \mathrm{T}\right.$ cells) and $\mathrm{OX} 40$ $\left(\mathrm{CD} 4^{+} \mathrm{T}\right.$ cells), when they recognize their cognate target antigen. $\mathrm{T}$ cells expressing the activation marker can then by purified using flow cytometry before their expansion and reinfusion into the tumor-bearing patient. 


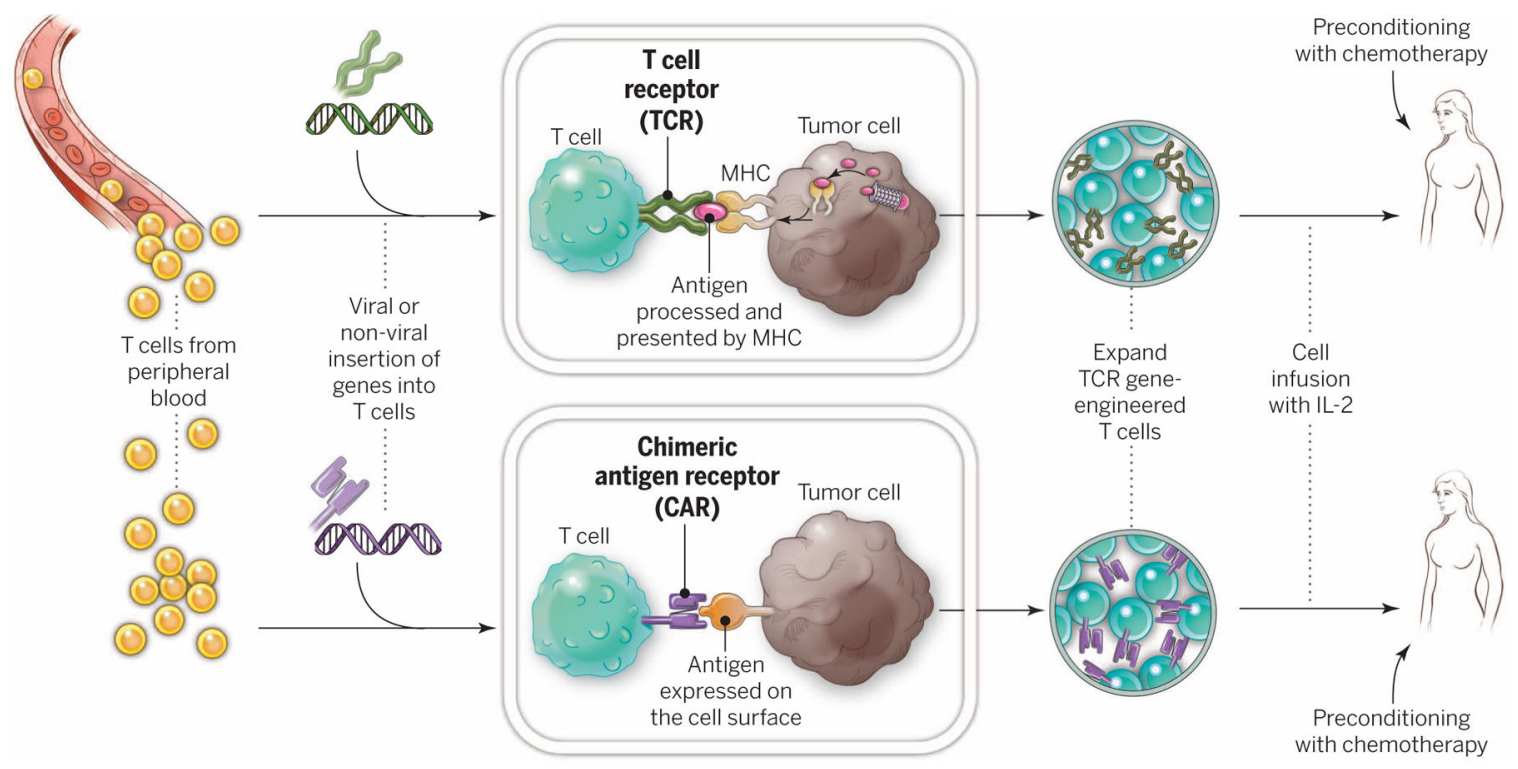

Fig. 4. Gene-modification of peripheral blood lymphocytes.

In an attempt to broaden the reach of ACT to other cancers, techniques are being developed to introduce antitumor receptors into normal $\mathrm{T}$ cells that could be used for therapy. The top panel shows the insertion of a conventional TCR into a patient's T lymphocytes, followed by the expansion and infusion back into the patient. The bottom panel shows the insertion of a CAR into a patient's T cell, followed by the expansion of these cells and their re-infusion. TCRs and CARs are fundamentally different in their structures and in the structures that they recognize. TCRs are composed of one a chain and one $\beta$ chain, and they recognize antigens that have been processed and presented by one of the patient's own MHC molecules. CARs are artificial receptors that can be constructed by linking the variable regions of the antibody heavy and light chains to intracellular signaling chains (such as CD3-zeta, CD28, 41BB) alone or in combination with other signaling moieties. CARs recognize antigens that do not need to be MHC-restricted, but they must be presented on the tumor cell surface. 


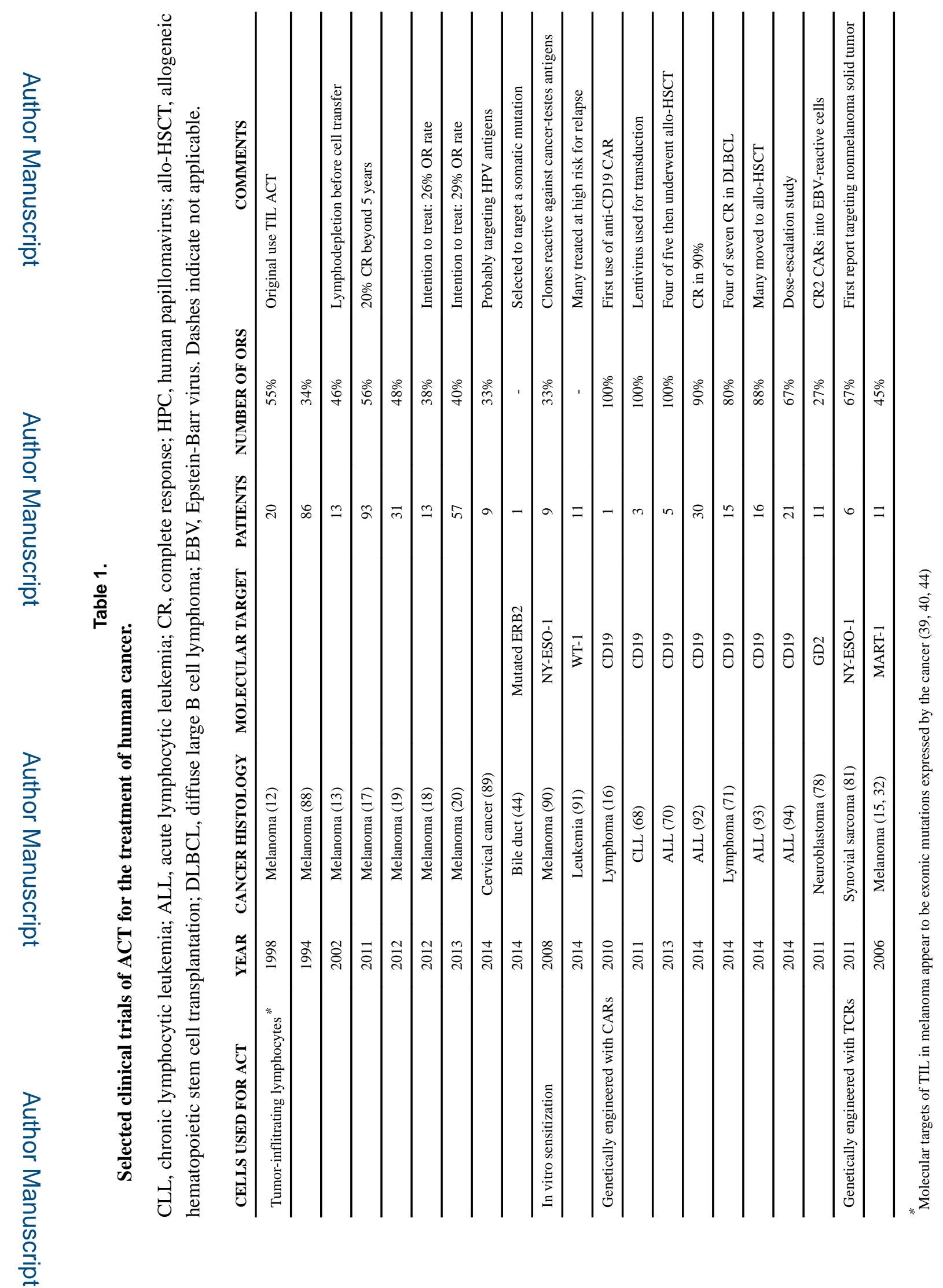

Science. Author manuscript; available in PMC 2018 December 17. 